\title{
Anatomical connection strength predicts dopaminergic drug effects on fronto-striatal function
}

\author{
Martine R. van Schouwenburg • Marcel P. Zwiers • \\ Marieke E. van der Schaaf • Dirk E. M. Geurts • \\ Arnt F. A. Schellekens • Jan K. Buitelaar • \\ Robbert J. Verkes • Roshan Cools
}

Received: 16 October 2012 / Accepted: 9 January 2013 /Published online: 13 February 2013

(C) The Author(s) 2013. This article is published with open access at Springerlink.com

\begin{abstract}
Rationale The neurotransmitter dopamine plays a key role in cognitive functions that are associated with fronto-striatal circuitry and has been implicated in many neuropsychiatric disorders. However, there is a large variability in the direction and extent of dopaminergic drug effects across individuals.

Objectives We investigated whether individual differences in dopaminergic drug effects on human fronto-striatal functioning are associated with individual differences in white matter tracts.

Methods The effects of the dopamine receptor agonist bromocriptine were assessed using functional magnetic resonance imaging in 22 healthy volunteers in a placebocontrolled, double-blind, within-subject design. Human psychopharmacology and functional neuroimaging were combined with functional connectivity analyses and structural connectivity analyses to establish a link between dopaminergic drug effects on fronto-striatal function and frontostriatal anatomy.
\end{abstract}

Electronic supplementary material The online version of this article (doi:10.1007/s00213-013-3000-5) contains supplementary material, which is available to authorized users.

M. R. van Schouwenburg $(\bowtie) \cdot$ M. P. Zwiers ·

M. E. van der Schaaf · D. E. M. Geurts · J. K. Buitelaar · R. Cools

Centre for Cognitive Neuroimaging, Donders Institute

for Brain, Cognition and Behaviour, Radboud University

Nijmegen, Kapittelweg 29,

6500 HB Nijmegen, The Netherlands

e-mail: m.vanschouwenburg@donders.ru.nl

M. R. van Schouwenburg • M. P. Zwiers $\cdot$ M. E. van der Schaaf $•$ D. E. M. Geurts · A. F. A. Schellekens • R. J. Verkes $\cdot$ R. Cools Department of Psychiatry, Radboud University Nijmegen Medical Centre, 6500 HB Nijmegen, The Netherlands
Results We demonstrate that bromocriptine alters functional signals associated with attention switching in the basal ganglia. Crucially, individual differences in the drug's effect on these signals could be predicted from individual differences in fronto-striato-thalamic white matter tracts, as indexed by diffusion tensor imaging. Anatomical frontostriatal connectivity also predicted drug effects on switchrelated functional connectivity between the basal ganglia and the prefrontal cortex.

Conclusions These data reinforce the link between dopamine, cognition and the basal ganglia and have implications for the individual tailoring of dopaminergic drug therapy based on anatomical fronto-striatal connection strength.

Keywords Prefrontal cortex - Attention · Basal ganglia . Connectivity $\cdot$ Dopamine $\cdot$ fMRI

\section{Introduction}

Dopamine is implicated in many neuropsychiatric disorders, such as schizophrenia, attention deficit hyperactivity disorder and addiction. These disorders are invariably accompanied by cognitive and attention deficits. Therefore, a better understanding of the mechanisms by which dopamine affects cognition and attention is crucial. However, individual variability poses a major challenge for dopaminergic drug research. While dopaminergic drugs improve cognitive function in some individuals, these drugs can impair cognitive function in others. Hence, the isolation of dopaminergic drug effects requires us to take into account such individual variability (Cools et al. 2007, 2009; Kimberg et al. 1997; Mehta and Riedel 2006; Wallace et al. 2011). Here, we investigated whether individual differences in dopaminergic 
drug effects on human cognitive processing could be predicted based on individual differences in white matter tracts.

Dopamine acts primarily as a neuromodulator, potentiating or attenuating synaptic transmission of classical neurotransmitters (Seamans and Yang 2004). At the level of the striatum, it stimulates dopaminergic receptors that are located near glutamatergic fronto-striatal and thalamo-striatal synapses, thus modulating information flow through fronto-striato-thalamo-frontal loops (Moss and Bolam 2010). One corollary of this observation is that dopamine's functional effects might vary as a function of fronto-striato-thalamo-frontal anatomical infrastructure. Specifically, dopaminergic drug effects might depend on individual differences in frontostriatal and/or thalamo-striatal connections. We used pharmacological functional magnetic resonance imaging (fMRI) to assess the effects of the dopamine receptor agonist bromocriptine on fronto-striatal activity during attention switching, as a function of anatomical connectivity as measured with diffusion tensor imaging. We anticipated that individual differences in dopaminergic drug effects on fronto-striatal function would depend on fronto-striato-thalamic anatomical connectivity.

This approach also enabled us to assess the pervasive but untested hypothesis that dopamine can alter prefrontal function indirectly by acting on the basal ganglia and by modulating information flow through fronto-striato-thalamofrontal circuitry (Hazy et al. 2007). So far, most studies have emphasized the role of the prefrontal cortex in dopamine's effects on cognition and attention. They demonstrate that dopamine can act on the prefrontal cortex to regulate attentional control (Noudoost and Moore 2011). However, dopamine receptors are particularly abundant in the basal ganglia; accordingly, dopamine might also act on the basal ganglia to modulate attentional control. This hypothesis is supported by a number of observations. First, pharmacological fMRI studies in humans have shown that dopamine modulates functional signals in the basal ganglia during a range of paradigms that require attention switching, such as task switching (Aarts et al. 2010), updating of working memory (Cools et al. 2007) and reversal learning (e.g. Dodds et al. 2008). Second, attentional control is disturbed in Parkinson's disease, a disorder that is characterized by relatively selective dopamine depletion in the basal ganglia. Third, dopamine has been shown to modulate frontostriatal functional connectivity in humans as well as in animals (Goto and Grace 2005; Honey et al. 2003; Nagano-Saito et al. 2008; Stelzel et al. 2010), in line with the hypothesis that dopamine might modulate the information flow through fronto-striato-thalamo-frontal circuits (Alexander et al. 1986).

The present study contributes to existing literature by investigating directly the link between dopaminergic drug effects on the basal ganglia, anatomical fronto-striatal connectivity and functional fronto-striatal connectivity.

\section{Methods and materials}

Subjects

Twenty-eight healthy volunteers participated in this study. Five subjects were excluded due to excessive movement in the scanner (more than two times the voxel size, e.g. translation $>6 \mathrm{~mm}$ ), data acquisition problems or image preprocessing problems. One subject had less than 10 switch trials and therefore was excluded. Accordingly, data are reported from 22 subjects (11 males, age [mean \pm SEM] $21.3 \pm 0.4$ ).

During an initial intake session, participants were screened by a medical doctor and a research nurse to exclude (a history of) psychiatric diseases, medical illness, substance abuse or a family history of psychiatric diseases (Supplementary Materials). In addition, the attention switching paradigm was practised in the MR scanner, while we obtained a structural scan and a diffusion-weighted scan.

All subjects gave written informed consent and were compensated for participation. The study was approved by the local ethics committee (CMO region Arnhem/Nijmegen, The Netherlands).

\section{Pharmacological design and procedures}

Subjects were tested on two occasions, once on placebo and once after intake of a single oral dose of the dopamine receptor agonist bromocriptine $(1.25 \mathrm{mg})$. The order of administration was randomized according to a counterbalanced, placebocontrolled and double-blind design (11 subjects received bromocriptine first, 11 subjects received placebo first). Timing of dosing was optimized for detecting drug effects during the fMRI scan which started 110 min after drug intake. Subjects were instructed to abstain from alcohol $24 \mathrm{~h}$ before drug intake and were not allowed to smoke or drink any caffeinated drinks on the day of testing. Blood samples, Bond and Lader visual analogue mood scales (Bond and Lader 1974) and measures of heart rate and blood pressure were obtained throughout the day. In addition, background neuropsychological tests (block completion, number cancellation, verbal fluency and digit span) were assessed at the end of the day (Supplementary Materials).

\section{Paradigm}

An attention switching paradigm was employed in which subjects switched attention when they detected a change in the stimulus exemplars of a non-selected category of face/scene stimuli. It was developed as an ecologically valid 
model of attention switching, because attention switches in real are often triggered by stimuli in a currently unattended stream of information. We have shown previously that this paradigm reliably recruits a fronto-striatal network, and it is therefore well suited to assess the effects of dopaminergic drugs on fronto-striatal functioning (van Schouwenburg et al. 2010). Subjects were presented with a series of stimulus pairs, each consisting of a superimposed face exemplar and scene exemplar (Fig. 1a). Subjects were instructed to select one of four exemplars by making a left or right response, depending on the location of the exemplar of their choice. This exemplar was then set as the correct stimulus, and subjects were instructed to continue selecting that stimulus on subsequent trials. After a variable number of correct trials, exemplars of the ignored category were replaced with novel exemplars. Subjects were instructed to switch attention to this other category and to choose one of the two novel exemplars, as soon as they detected a change. Trials on which novel exemplars were introduced and on which subjects detected the change and switched to one of the novel exemplars where classified as switch trials (Fig. 1c). On some trials, subjects failed to detect the novel exemplars and kept responding to the previously correct exemplar (non-switch trials). In this case, negative feedback was presented, usually leading subjects to switch on the subsequent trial. Trials on which no novel stimuli were introduced were defined as repeat trials (Fig. 1b). Four subjects had less that 10 non-switch trials on one of two sessions. Therefore, we focused all analyses on switch and repeat trials.
Subjects were presented with an average of 349 trials (SEM 5), on which novel exemplars were introduced on 82 trials. The sequence of the faces and scenes presented was randomized across subjects but was constant within subjects across the two sessions. For more details on the paradigm, see van Schouwenburg et al. (2010).

\section{Behavioural analysis}

Behavioural analysis focused on the switch likelihood, which was calculated as the percentage of immediate switches in response to a novel stimulus, and reaction time analyses. Excluded from reaction time analyses were the first trial of each block, all trials on which subjects received negative feedback and trials following negative feedback. For each subject, we calculated the median reaction time, rather than mean reaction time, to minimize the influence of outliers. Next, we calculated the mean reaction times \pm SEM across subjects. Planned contrasts were assessed using repeated-measures ANOVAs or paired sample $t$ tests. The statistical threshold was set at $p<0.05$ (two-tailed).

\section{fMRI data acquisition and analysis}

Whole-brain functional images and a T1-weighted anatomical scan were obtained on a Siemens 3-T MR scanner (for scanning parameters, see Supplementary Materials). Massunivariate data analysis was performed using SPM5 software (Wellcome Trust Centre for Neuroimaging, London,

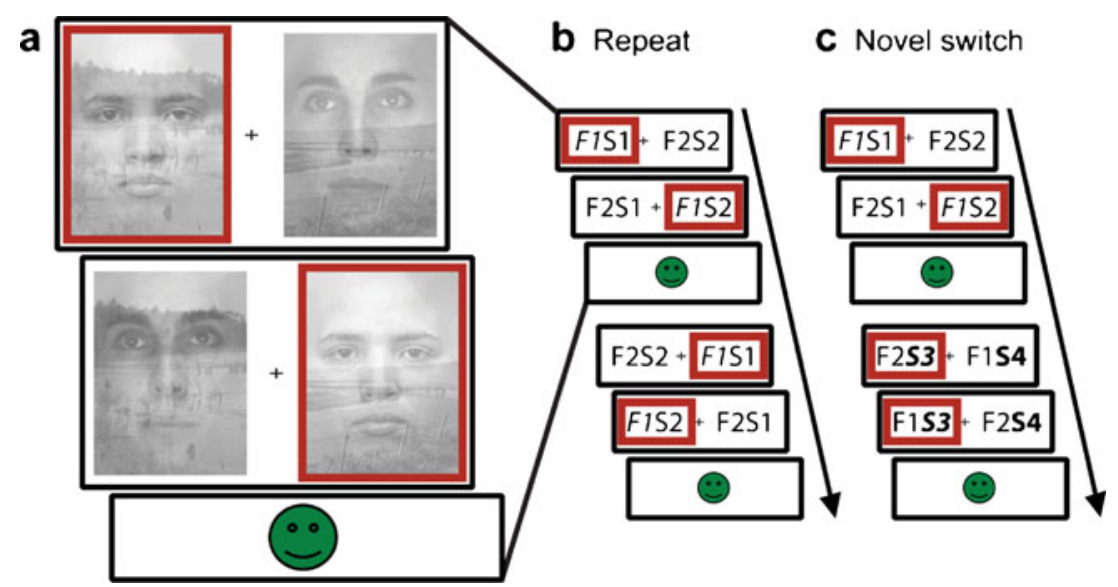

Fig. 1 Subjects were instructed to select one stimulus exemplar (left versus right) within one category (faces versus scenes) and to keep selecting that same exemplar for a number of trials, until novel exemplars were introduced in the non-selected category, requiring a switch in attention. a Stimuli consisted of superimposed exemplars of a face and a scene. Stimulus pairs were presented twice within each trial and the combination of face and scene was reversed on the second presentation relative to the first, enabling us to identify the attended stimulus. Feedback was presented after each trial and was positive only if the subject selected the correct stimulus twice within the trial. Red boxes indicate a possible response sequence. b, $\mathbf{c}$ Two consecutive trials constituting our two trial types of interest. The stimuli are displayed schematically for illustrative purposes $(F 1$, face $1 ; S 1$, scene $1 ; F 2$, face 2; $S 2$, scene 2). Attended stimuli are displayed in italic font. b Repeat trial: on the first trial, the subject attends to F1. On the next trial, no novel stimuli are introduced and the subject keeps attending to F1. The second trial is thus defined as a repeat trial. c Switch trial: The subject attends to F1 on the first trial. On the second trial, novel stimuli of the non-selected category, in this case scenes, are introduced ( $S 3$ and $S 4$ ). The subject detects this change and switches attention to one of two novel stimuli (here $S 3$ ) 
UK). Preprocessing procedures of functional images were performed as described previously (van Schouwenburg et al. 2010).

In a general linear model (GLM), we included two regressors of interest: switch and repeat trials. In addition, we modelled non-switch trials (regressor 3), trials following non-switch trials (regressor 4), all error trials, missed trials and trials after an error or after a missed trial (regressor 5), and the six realignment parameters (regressors 6-11) as regressors of no interest. All paradigm-related regressors were modelled as delta functions at the onset of the first stimulus-pair presentation within a trial and were convolved with a canonical haemodynamic response function (hrf) including time derivatives. Time series were high-pass filtered (128 s).

Parameter estimates for the regressors of interest were estimated at the first level, for each session separately, and then used in a second level random effects $2 \times 2$ factorial design with the within-subject factors trial type (switch and repeat) and drug (placebo and bromocriptine).

To assess drug effects in the basal ganglia, we defined a volume of interest (VOI) as a sphere with a 6$\mathrm{mm}$ radius (size of the applied smoothing kernel) around the peak voxel for the switch versus repeat contrast averaged across drug conditions within the left (MNI coordinates, -1220 ) and right (14 20 0) basal ganglia [defined as the caudate, putamen and pallidum, according to the automated anatomical labelling (AAL) interface (Tzourio-Mazoyer et al. 2002)]. (Similar results were obtained when definition of the VOI was based on clusters in the basal ganglia showing an increase for switch versus repeat, see Supplementary Material). The left and right VOIs were then combined. Definition of VOIs and VOI data extraction were done using MarsBaR (Brett et al. 2002). Note that the numbers of switch and repeat trials were matched between drug conditions. This allowed us to define a VOI based on the switch minus repeat contrast across drug conditions without introducing a bias towards finding a drug effect (Kriegeskorte et al. 2010).

The main effects of task (switch versus repeat) were tested and displayed at a threshold of $p<0.05$ familywise error (FWE)-corrected for the whole brain $\left(p_{\mathrm{FWE}}\right)$.

Drug effects were assessed at the voxel level, corrected for multiple comparisons across our VOI in the basal ganglia (defined functionally, as described above) $\left(p_{\text {svc }}<0.05\right)$. In addition, exploratory analyses were performed across the whole brain $\left(p_{\mathrm{FWE}}<0.05\right)$.

Figures were displayed using MRIcroN (Rorden et al. 2007). SPMs were superimposed on a skull-stripped template in MNI space, unless indicated otherwise.
Diffusion tensor imaging analysis

To determine anatomical connectivity, we acquired diffusion tensor images (DTI). Raw DTI data were preprocessed using an in-house software (Zwiers 2010). The DTI images were realigned and eddy current-corrected by residual error minimization of the diffusion tensor model (Andersson and Skare 2002). Susceptibilityinduced echo-planar imaging distortions were corrected by warping the images to the distortion-free $\mathrm{T} 1$ reference image (Studholme et al. 2000) using an in-house developed implementation (Visser et al. 2010).

Diffusion tensors were then robustly estimated using our artefact-insensitive compute algorithm (Zwiers 2010). Mean diffusivity (MD) and fractional anisotropy (FA) measures were computed from the diffusion tensor eigenvalues. FA and MD maps were normalized to the T1 ICBM template using the unified segmentation parameters of the structural image and spatially smoothed using a Gaussian kernel of 8-mm full width at half maximum.

The resulting FA and MD maps were then tested using a second-level one-sample $t$ test, with the drug effect on basal ganglia blood oxygenation leveldependent (BOLD) signal for the switch versus repeat contrast as a covariate. This drug effect was calculated for each subject separately by subtracting the average switch-related BOLD signal across the basal ganglia VOI on placebo from the average switch-related BOLD signal across the basal ganglia VOI on bromocriptine. FA results were masked by a threshold mask of FA $>0.2$.

Correlations between FA/MD values and drug effects on basal ganglia BOLD signal were assessed at the voxel level, corrected for multiple comparisons across the functionally defined VOI in the basal ganglia $\left(p_{\mathrm{svc}}<0.05\right)$. In addition, exploratory analyses were performed across the whole brain $\left(p_{\mathrm{FWE}}<0.05\right)$.

\section{Fibre tracking}

The FA region showing a significant correlation with the drug effect on basal ganglia BOLD signal was then used for probabilistic diffusion tractography to identify white matter tracts connecting with this location. More specifically, we defined a VOI as a sphere with a radius of $6 \mathrm{~mm}$ around the peak voxel of the correlation (MNI coordinates, 1860 ). For each subject, this VOI was brought back into native space, using the inverse of the computed normalization parameters. FMRIB's Diffusion Toolbox (part of FMRIB's Software Library) was used to build up distributions on diffusion parameters at each voxel, allowing for crossing fibres (Behrens et al. 2007) and subsequent probabilistic tracking from the VOI to all other voxels in the brain (using standard settings). To 
eliminate spurious connections, tractography in individual subjects was thresholded to include only voxels through which at least 50 samples had passed (out of 5,000). These individual tracts were then binarized and summed across subjects to produce group probability maps. In these maps, each voxel value represents the number of subjects in whom the pathway passes through that voxel. Results were thresholded to display only those paths that were present in at least $50 \%$ of the subjects (11 out of 22). A similar approach has been used previously (e.g. Boorman et al. 2007; Floel et al. 2009).

\section{Psychophysiological interaction analysis}

Functional connectivity was assessed using psychophysiological interaction (PPI) analysis (Friston et al. 1997). PPI works under the assumption that the degree to which the BOLD signal in one area can be predicted, based on BOLD signal in another, corresponds to the contribution of the second region to the first region. The PPI then assesses whether this contribution changes over experimental conditions. In other words, it assesses whether region A shows higher or lower connectivity with region $\mathrm{B}$, during condition C, compared with condition D. Time series were extracted from a seed voxel in the basal ganglia that showed an increase in BOLD signal during switching (versus repeat), for each subject individually. Because the exact locations of activation maxima varied across subjects, we determined the individual peak voxels in the basal ganglia, using the constraints that it (1) exceeded a threshold of $p<0.05$ (uncorrected) in the switch versus repeat contrast and (2) was within $6 \mathrm{~mm}$ of the group maximum (MNI coordinates, $160-2)$ of the drug effect on the switch versus repeat contrast. To summarize the regional time series, we computed the first eigenvector across all suprathreshold voxels ( $p<0.05$ uncorrected) within $3 \mathrm{~mm}$ of this peak voxel. The time series were then multiplied by a vector coding for the experimental conditions (switch versus repeat) to obtain the PPI.

On the subject level, we included the PPI as a regressor of interest in a GLM. The experimental conditions and the extracted time series were modelled as additional regressors, in order to assess the PPI estimates over and above shared functional activation and task-independent correlations in BOLD signal between the seed and other regions. This approach ensures that any obtained PPI results are independent of univariate results. These regressors were convolved with a canonical hrf and high-pass filtered (128 s). In addition, the six realignment parameters were modelled. The PPI analysis was performed for each session separately.

The difference between PPI maps on the drug and placebo session was calculated for each subject. These difference maps were then brought to the second random effects level in a one-sample $t$ test, with FA values as a covariate. These FA values were extracted from the region showing a significant correlation with the drug effect on switch-related BOLD signal in the basal ganglia (as shown in Fig. 4a).

Our functional connectivity analyses aimed to reveal that drug effects on the switch-related basal ganglia signal were accompanied by drug effects on functional connectivity between the basal ganglia and the prefrontal cortex. To this end, we focused our functional connectivity analyses on a prefrontal region which we know, based on our previous study with this task (van Schouwenburg et al. 2010), to be involved in attention switching: the right inferior frontal gyrus (IFG). Selection of this region is further justified based on other studies suggesting that this region plays an important role in the selective focusing of attention on currently relevant information (Gazzaley et al. 2004; Hampshire et al. 2007; Petrides and Pandya 2009). We combined subregions of the right IFG according to the AAL interface (Tzourio-Mazoyer et al. 2002) to obtain an anatomical VOI.

Drug effects were assessed at the voxel level, corrected for multiple comparisons across the VOI $\left(p_{\mathrm{svc}}<0.05\right)$. In addition, exploratory analyses were performed across the whole brain $\left(p_{\mathrm{FWE}}<0.05\right)$.

\section{Independence of analyses}

We would like to emphasize here that we are very well aware of the danger of double dipping and circular analyses (Kriegeskorte et al. 2009) and that the effects derived from the univariate fMRI analyses, the PPI analysis and the DTI/FA analyses are all independent of each other. In the following paragraph, we summarize our analysis steps and reiterate why they are not circular. First, the drug effect on switch-related BOLD signal was assessed within a basal ganglia region that responded to the main effect of switch versus repeat, collapsed across drug sessions. Thus, drug effects were calculated within a region that was selected from the same dataset. Nevertheless, this analysis is not biased because the main task contrast (switch versus repeat) and the interaction (drug $\times$ task) contrast are orthogonal (Friston et al. 2006). In addition, the actual main and interaction effects were also orthogonal, because the numbers of switch and repeat trials were matched between drug sessions (Kriegeskorte et al. 2009). In the second step, we calculated the correlation between FA values and the drug effect on switch-related BOLD signal. This was done within this same basal ganglia region of interest. This analysis is not biased, because the DTI and BOLD datasets are two separate, independent datasets. The third step was the PPI analysis, which was conducted using a seed region in the basal ganglia that was calculated based on the drug effect on switch-related BOLD signal. This analysis is also not 
biased, because the inclusion of seed and task-related regressors in the PPI model ensures that any obtained PPI results are over and above shared functional activation and task-independent correlations in BOLD signal between the seed and other regions. Thus, the PPI results are independent of the univariate fMRI results. Our final step was to calculate the correlation between FA values, extracted from the basal ganglia region that correlated with the drug effect on BOLD, and the PPI effects, which were calculated using a seed defined based on the drug effect on BOLD. Although perhaps more counterintuitive, it is critical to realize that this analysis is also not biased, for the same reason as mentioned above: PPI results are independent of univariate results.

\section{Results}

Neural responses during attention switching

Consistent with our previous study (van Schouwenburg et al. 2010), attention switching increased BOLD signal in a network of regions. These included the basal ganglia, IFG, thalamus, insula, hippocampus, anterior cingulate cortex/supplementary motor area, inferior and superior parietal cortex, visual association cortex and midbrain (Fig. 2, Table 1).

Dopaminergic drug effects during attention switching

To investigate the effects of dopamine on this attention switching network, we compared BOLD signal after administration of the dopamine $\mathrm{D}_{2}$ receptor agonist bromocriptine with BOLD signal after placebo. Based on prior work (Cools et al. 2007, 2009) and the distribution of $\mathrm{D}_{2}$ receptors in the brain, we expected bromocriptine to act on the basal ganglia. Consistent with this prediction, bromocriptine increased BOLD signal selectively in the task-active regions of the basal ganglia during the switch trials relative to the repeat trials $\left(t=3.91, p_{\mathrm{svc}}=\right.$ $0.005)$ (Fig. 3). No other effects were observed at $p_{\text {FWE }}$ $<0.05$ across the whole brain, although we report subthreshold effects (at $p_{\text {uncorrected }}<0.001$ ) in the Supplementary Materials for completeness (Table S2). This result supports previous findings that show the basal ganglia as the site of action of dopaminergic drug effects on attention switching (Cools et al. 2007; Dodds et al. 2008). However, large individual differences were observed in the degree to which bromocriptine modulated BOLD signal in this region during switching. While bromocriptine increased BOLD signal in some subjects, it decreased BOLD signal in others (Fig. 3b).

Correlations between drug effects and white matter structure

Next, we assessed whether these drug effects in the basal ganglia were associated with individual differences in the underlying white matter structure. To this end, we extracted BOLD signal from our functional basal ganglia VOI for each subject and calculated the difference between the bromocriptine session and the placebo session. These values were entered as a covariate in a secondlevel GLM to assess associations with whole-brain FA values, a measure of white matter integrity. This revealed a significant association between the drug effect on basal ganglia BOLD signal and FA values in a region located in the anterior limb of the capsula interna $(t=4.86$, $p_{\mathrm{svc}}=0.003$ ) (Fig. 4a). This region was immediately adjacent to the region in which bromocriptine exerted its effect (Fig. 4c). The association was negative, such that bromocriptine enhanced BOLD signal in the basal ganglia of subjects with low local white matter integrity while decreasing BOLD signal in the basal ganglia of subjects with high local white matter integrity (Fig. 4b). There were no other effects at $p_{\mathrm{FWE}}<0.05$ across the whole brain. We report subthreshold effects at $p_{\text {uncorrected }}$ $<0.001$ in the Supplementary Materials for completeness (Table S3).

\section{Probabilistic diffusion tractography}

To identify the white matter tracts connecting with the region in which the drug-FA association was found, we used the cluster found in the capsula interna as a seed region for probabilistic diffusion tractography (Behrens et al.
Fig. 2 Statistical parametric map of the main effect of attention switching (switch versus repeat), across drug sessions (unmasked). The bar indicates $t$ values, and the figures are thresholded for a $t$ value of 5.10, corresponding to a $p$ value of 0.05 FWEcorrected for multiple comparisons
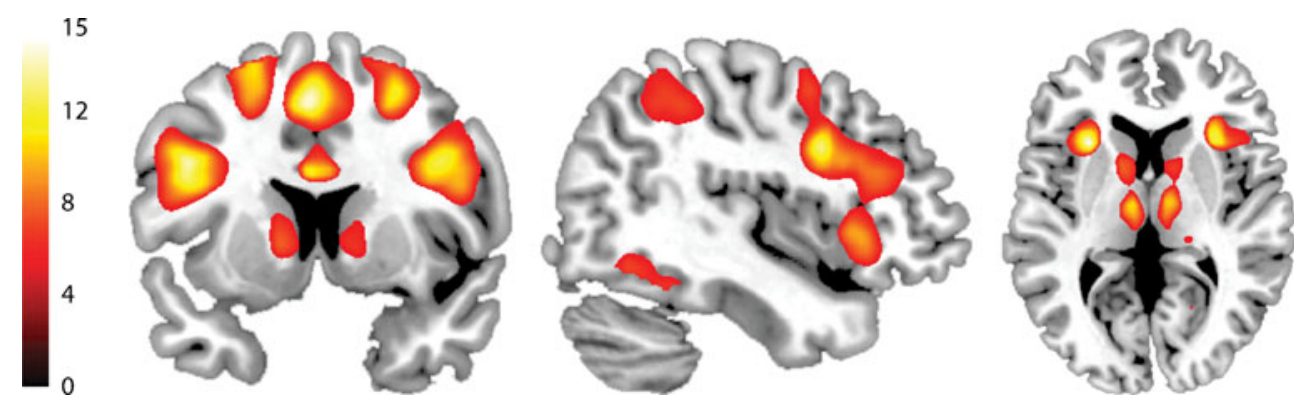
Table 1 Main effects of task (switch versus repeat), $p<0.05$ FWE-corrected, with a contiguous voxel cluster threshold $k>5$. Peak voxels were localized using the SPM Anatomy Toolbox (Eickhoff et al. 2007)

\begin{tabular}{|c|c|c|c|c|c|}
\hline \multirow[t]{2}{*}{ Region } & \multirow[t]{2}{*}{ Cluster size } & \multicolumn{3}{|c|}{ Local maximum } & \multirow{2}{*}{$\begin{array}{l}\text { Cluster } \\
\text { statistics } \\
t \text { value }\end{array}$} \\
\hline & & $x$ & $y$ & $z$ & \\
\hline Insula & 755 & -32 & 22 & 0 & 17.40 \\
\hline Supplementary motor area/anterior cingulate cortex & 2,590 & -4 & 18 & 44 & 17.13 \\
\hline Inferior frontal gyrus & 1,571 & -42 & 6 & 30 & 15.49 \\
\hline Insula/inferior frontal gyrus & 3,355 & 32 & 26 & 2 & 14.43 \\
\hline Midbrain/thalamus/basal ganglia & 2,425 & -6 & -28 & -6 & 12.25 \\
\hline Middle/superior frontal gyrus & 861 & -24 & 0 & 54 & 11.92 \\
\hline Inferior/superior parietal lobule & 1,881 & 34 & -46 & 46 & 11.73 \\
\hline Inferior/superior parietal lobule & 2,497 & -46 & -40 & 42 & 11.35 \\
\hline Inferior temporal gyrus & 72 & -44 & -58 & -8 & 9.30 \\
\hline Inferior temporal gyrus/fusiform gyrus & 351 & 30 & -34 & -20 & 7.32 \\
\hline Posterior cingulate cortex & 62 & -4 & -28 & 28 & 6.96 \\
\hline Fusiform gyrus & 30 & -28 & -54 & -10 & 6.29 \\
\hline Middle frontal gyrus & 6 & -32 & 52 & 24 & 5.65 \\
\hline Calcarine gyrus & 7 & -12 & -76 & 8 & 5.65 \\
\hline Precuneus & 9 & 22 & -56 & 22 & 5.41 \\
\hline
\end{tabular}

2007). White matter fibres were revealed in a fronto-striatothalamic tract, running from the basal ganglia to the IFG and from the basal ganglia to the thalamus, extending into the midbrain (Fig. 5a).

Drug effects on functional connectivity

We predicted that dopamine would also alter functional connectivity between the prefrontal cortex and basal ganglia. Thus, next we investigated whether drug effects on functional (switch-related) connectivity between the basal ganglia and right IFG also depended on white matter integrity.

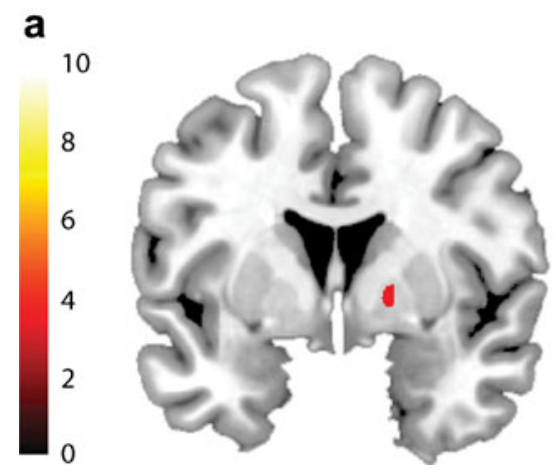

Fig. 3 a Whole-brain statistical parametric map of drug effects on switch-related BOLD signal. The statistical parametric map is masked by the main effect of attention switching (thresholded at $p<0.001$ uncorrected). The bar indicates $t$ values of the drug effect (bromocriptine-placebo), and the figure is thresholded for a $t$ value of 3.19,
In line with our predictions, whole-brain analyses revealed that FA values associated with the drug effect on the basal ganglia were also associated with the drug effect on functional connectivity between this region and the right IFG (MNI coordinates, $482422 ; t=6.17 ; p_{\text {svc }}=0.009$ ). The cluster was localized close to the endpoint of the anatomical fronto-striato-thalamic tract revealed by our tractography analyses (Fig. 5b). Thus, consistent with our prediction, dopaminergic drug effects on the basal ganglia during attention switching were accompanied by dopaminergic drug effects on functional fronto-striatal connectivity, in an anatomy-dependent manner. Note that we did not find a main effect of drug on functional connectivity, that is, when white matter integrity was not taken into account.

b

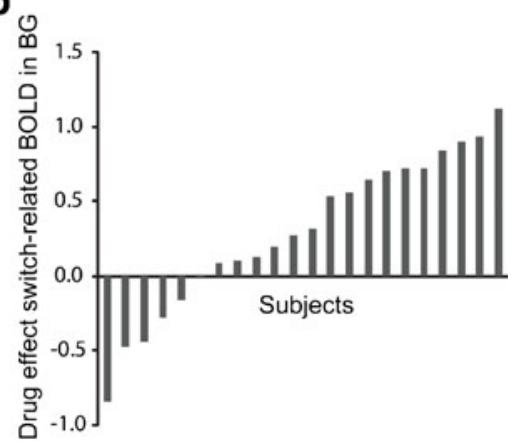

corresponding to a $p$ value of 0.001 uncorrected for multiple comparisons. b Shown are individual differences in drug effects on switchrelated BOLD signal from the basal ganglia, extracted from the cluster displayed in $\mathbf{a}$ 
Fig. 4 a Whole-brain statistical parametric map (unmasked) of association between fractional anisotropy and drug effect on switch-related basal ganglia (BG) BOLD signal,

superimposed on the mean fractional anisotropy image from all participants. The bar indicates $t$ values, and the figure is thresholded for a $t$ value of 3.55 , corresponding to a $p$ value of 0.001 uncorrected for multiple comparisons. b Data were extracted from the correlated cluster and plotted for illustration purposes. c Overlap between drug effect on switch-related basal ganglia BOLD signal (green) and its association with fractional anisotropy (red) a
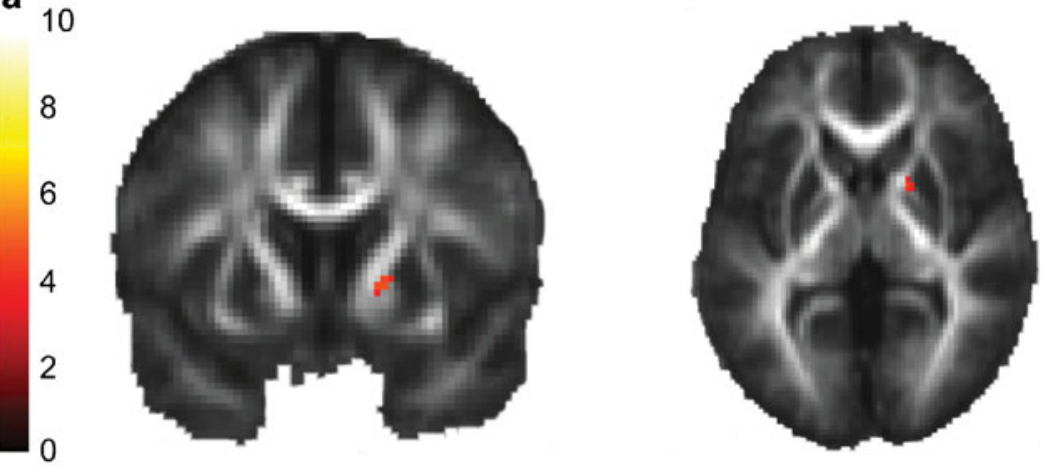

b

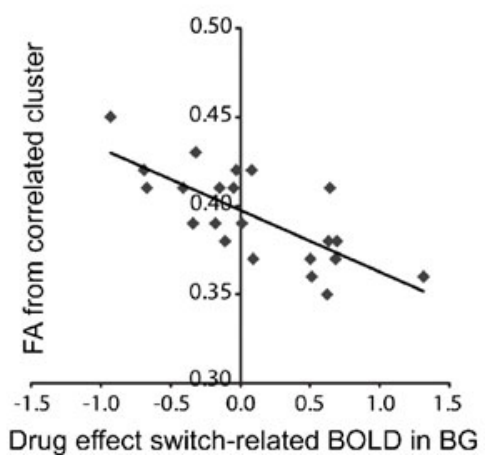

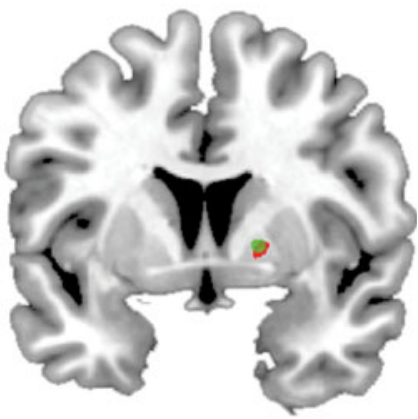

Additional analyses

A number of additional analyses were performed to exclude possible confounding factors. Using voxel-based morphometry analyses, we excluded the possibility that our white matter correlations were in fact driven by differences in grey matter. Moreover, while we found an association between the drug effect on switch-related basal ganglia BOLD signal with FA, no such association was found with MD. This indicates that our results cannot be explained in terms of differences in cell density (as measured with MD). Instead, our results likely reflect directional (axonal) organization (as measured with FA). For more details on these supplementary analyses, see Supplementary Materials. a

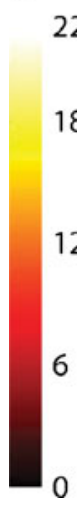

b
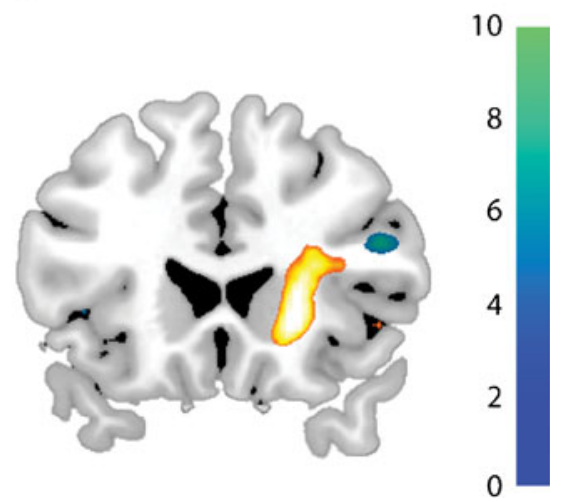

on functional connectivity from a basal ganglia seed, as assessed by PPI (blue/green), as a function of individual differences in FA. The bar indicates $t$ values, and the figure is thresholded for a $t$ value of 3.55 , corresponding to a $p$ value of 0.001 uncorrected for multiple comparisons. White matter tracts derived from probabilistic tractography are displayed in yellow 
Behavioural analyses

Reaction times were significantly slower on switch trials compared with repeat trials $\left(F_{1,21}=107.0, p<0.0001\right)$. There was no main effect of drug $\left(F_{1,21}=1.4, p=0.2\right)$ or an interaction between drug and trial type $\left(F_{1,21}=1.1, p=0.3\right)$ (RT placebo: switch, 1,030 \pm 53 ; repeat, $746 \pm 36$ ) (RT bromocriptine: switch, 1,098 \pm 73 ; repeat, $774 \pm 44$ ).

Bromocriptine also had no effect on switch likelihood. Mean switch likelihood was not significantly different between the placebo session $(67.2 \pm 4.1 \%)$ and the bromocriptine session $(64.7 \pm 3.9 \%)\left(t_{1,21}=1.2, p=0.3\right)$, also not when individual differences in FA were taken into account. There were no brain-behaviour associations.

Drug effects on physiological measurements, neuropsychological tests and mood ratings

Bromocriptine significantly decreased prolactin levels and systolic and diastolic blood pressure, indicating that bromocriptine reached the systemic circulation. Drug effects were not attributable to non-specific drug effects on mood and global cognitive functioning, as bromocriptine did not affect general cognitive performance or mood, in line with previous studies (Cools et al. 2007; van der Schaaf et al. 2012) (see Supplementary Materials).

\section{Discussion}

Dopaminergic drug effects vary greatly between individuals such that the same drug can exert effects in opposite directions. Our study establishes an important new link between dopaminergic drug effects on fronto-striatal function and white matter integrity of anatomical fronto-striato-thalamic connections. More specifically, we found that the effect of bromocriptine on functional signals in the basal ganglia and fronto-striatal connectivity was significantly associated with anatomical fronto-striato-thalamic connectivity. Bromocriptine had diametrically opposite effects in subjects with high and low white matter tract integrity, as indexed by fractional anisotropy values. Fractional anisotropy relies on several microstructural properties of white matter tissue, such as the level of axon myelination, intact axonal membranes, fibre density and fibre diameter (Beaulieu 2002). This suggests that bromocriptine had opposite effects as a function of neuronal communication efficiency, in line with the observation that dopamine acts as a neuromodulator (Moss and Bolam 2010).

Our approach resembles that used previously to link anatomical connectivity with individual differences in behaviour (Forstmann et al. 2008; Tuch et al. 2005) and functional connectivity (Boorman et al. 2007; Neubert et al. 2010). For example, it was shown that individual differences in response inhibition performance depended on fractional anisotropy in the IFG (Forstmann et al. 2008). However, to our knowledge, no previous work has revealed associations between neurochemical (drug) effects and anatomical connectivity. This finding should have important implications for neuropsychiatric drug treatment. For example, taking into account white matter integrity might contribute to individual tailoring and thus optimization of drug treatment strategies in dopaminerelated neuropsychiatric disorders.

The finding that bromocriptine had diametrically opposite effects as a function of anatomy is reminiscent of the invertedU-shaped relationship between dopamine and cognitive function (Cools et al. 2007; Kimberg et al. 1997). Dopaminergic drugs are beneficial for some subjects, while impairing functioning in others. In line with this, we found no main effect of drug on functional connectivity. However, when taken into account anatomical connectivity, these individual differences could be explained. So far, individual differences in dopaminergic drug effects have not been linked to brain structure. Previous studies found individual differences in dopaminergic drug effects to depend on baseline working memory capacity, presumably reflecting individual differences in baseline dopamine levels as a consequence of genetic predisposition (Cools and D'Esposito 2011; Cools et al. 2009). We did not find an association between drug effects and baseline working memory capacity (as measured with listening span). An important aim for future work is to assess how baseline neurochemical, genetic and anatomical factors interact to determine drug efficacy.

The drug-FA association, revealed by whole-brain analysis, was remarkably regionally selective. Indeed, the effect was restricted to a region in the capsula interna transversing the basal ganglia, immediately adjacent to the region that was modulated by the drug. Probabilistic tractography revealed that this white matter region projected to the IFG, and the thalamus, extending into the midbrain. Furthermore, individual differences in white matter integrity predicted drug effects on functional connectivity between the basal ganglia and an inferior frontal cluster that was located right next to the inferior frontal extension of these tracts. These findings are in line with the suggestions that dopamine acts on the basal ganglia to alter information flow through anatomical fronto-striatal-thalamic circuits.

We and others have shown previously that dopamine can modulate cognitive BOLD signal in the basal ganglia, particularly during tasks that require cognitive flexibility (Cools et al. 2007; Dodds et al. 2008; Jocham et al. 2009). Following these observations, we focused our analyses on the basal ganglia, where we found a main effect of drug in line with these previous studies. We would like to point out here that fMRI does not allow us to assess whether the observed drug effects are a direct effect of drugs acting on the basal ganglia or secondary from effects elsewhere. For example, previous studies showed that dopamine modulates cognitive BOLD 
signal in the prefrontal cortex (Gibbs and D'Esposito 2005a, b) as well as functional connectivity between the basal ganglia and the prefrontal cortex (Krugel et al. 2009; Nagano-Saito et al. 2008; Stelzel et al. 2010; Wallace et al. 2011). As a consequence of the strong anatomical (and functional) connectivity within the fronto-striatal network, dopamine might modulate this network as a whole, rather than single regions within this network. The present work substantiates this idea by showing that the link between drug effects on functional fronto-striatal connectivity is mediated by anatomical connectivity between the basal ganglia and the prefrontal cortex.

The present study investigated the effect of bromocriptine on a task that required a switch in attention in response to novel stimuli. Such switching depends on multiple subcomponent processes, including novelty detection, inhibition of a previous stimulus-response association, and selection of a novel stimulus. Here, we did not aim to isolate the specific subcomponent process that was affected by the drug, but rather we aimed to test drug effects on an ecologically valid model of attention switching. Nevertheless, it is notable that the regions within the functional network that were affected by bromocriptine, in particular the IFG and the basal ganglia, have previously been associated with inhibition (Aron et al. 2007; Duann et al. 2009; Zandbelt and Vink 2010). Similarly, the thalamus has been implicated in the inhibition of ongoing behaviour and action selection. More specifically, Ding et al. have proposed that salient signals that are detected by the thalamus are transmitted to the striatum via thalamo-striatal axonal connections, where they elicit a characteristic firing pattern that is optimized for halting ongoing (motor and cognitive) programmes (Ding et al. 2010). Their study revealed that activation of thalamo-striatal axons mimicked the response to salient stimuli in the sense that it induced burst firing of striatal interneurons, which in turn triggered prolonged enhancement of postsynaptic responsiveness of striatopallidal neurons. Critically, the effect of stimulating the thalamus on striatal interneurons depended on $\mathrm{D}_{2}$ receptor stimulation. Accordingly, they proposed that effects of $\mathrm{D}_{2}$ receptor agents on attention switching to salient events might depend on axonal connections that enable thalamic gating of striatopallidal signals (Ding et al. 2010). The findings of the present study concur with this hypothesis. Thus, one mechanism by which dopamine $\mathrm{D}_{2}$ receptor stimulation might modulate attention switching to salient events is by affecting thalamic signals to the striatum and subsequent flow through fronto-striatal circuitry.

One important caveat of the present study is that we did not find any drug effects on the behavioural measures of our task. This was unexpected but might be accounted for by masking of a subtle behavioural effect by noise induced by the scanner environment. Perhaps, our measure of behaviour was not sufficiently sensitive and thus masked by such non-specific effects. Indeed, in a recent study in which subjects performed the same attention switching paradigm outside the scanner, we found that behavioural performance on the task was correlated with FA values in the exact same basal ganglia region (van Schouwenburg, Onnink, ter Huurne, Kan, Zwiers, Hoogman, Franke, Buitelaar and Cools, Attention switching depends on white matter integrity of the basal ganglia: a study in adults with ADHD, unpublished data). Nevertheless, the lack of a behavioural effect in the current study does ensure that the observed neural drug effects are not confounded by drug-related differences in performance. Thus, the present study establishes a strong link between dopamine's effects on the basal ganglia, functional fronto-striatal connectivity during attention switching and fronto-striato-thalamic anatomy. Future work should aim to extend the link between fronto-striatal anatomy and dopaminergic drug effects on fronto-striatal function to behaviour.

Acknowledgments We thank Jan Leytens for the assistance with medical screening of the subjects and the Chemical Endocrinology Department at the Radboud University Nijmegen, especially Fred Sweep and Rob van den Berg, for analyzing the blood samples. This work was supported by a VIDI grant from the Innovational Research Incentives Scheme of the Netherlands Organisation for Scientific Research (NWO) to RC and a Human Frontiers Science Program grant to Kae Nakamura, Nathaniel Daw and RC.

Open Access This article is distributed under the terms of the Creative Commons Attribution License which permits any use, distribution, and reproduction in any medium, provided the original author(s) and the source are credited.

\section{References}

Aarts E, Roelofs A, Franke B, Rijpkema M, Fernandez G, Helmich RC, Cools R (2010) Striatal dopamine mediates the interface between motivational and cognitive control in humans: evidence from genetic imaging. Neuropsychopharmacology 35:1943-1951

Alexander GE, DeLong MR, Strick PL (1986) Parallel organization of functionally segregated circuits linking basal ganglia and cortex. Annu Rev Neurosci 9:357-381

Andersson JL, Skare S (2002) A model-based method for retrospective correction of geometric distortions in diffusion-weighted EPI. NeuroImage 16:177-199

Aron AR, Behrens TE, Smith S, Frank MJ, Poldrack RA (2007) Triangulating a cognitive control network using diffusion-weighted magnetic resonance imaging (MRI) and functional MRI. J Neurosci $27: 3743-3752$

Beaulieu C (2002) The basis of anisotropic water diffusion in the nervous system - a technical review. NMR Biomed 15:435-455

Behrens TE, Berg HJ, Jbabdi S, Rushworth MF, Woolrich MW (2007) Probabilistic diffusion tractography with multiple fibre orientations: what can we gain? NeuroImage 34:144-155

Bond A, Lader M (1974) Use of analog scales in rating subjective feelings. Brit J Med Psychol 47:211-218

Boorman ED, O'Shea J, Sebastian C, Rushworth MF, Johansen-Berg $\mathrm{H}$ (2007) Individual differences in white-matter microstructure reflect variation in functional connectivity during choice. Curr Biol 17:1426-1431

Brett M, Anton JL, Valabregue R, Poline JB (2002) Region of interest analysis using an SPM toolbox [abstract]. Presented at the 8 th international conference on functional mapping of the human brain, Sendai, Japan 
Cools R, D’Esposito M (2011) Inverted-U-shaped dopamine actions on human working memory and cognitive control. Biol Psychiatry 69:e113-e125

Cools R, Sheridan M, Jacobs E, D'Esposito M (2007) Impulsive personality predicts dopamine-dependent changes in frontostriatal activity during component processes of working memory. J Neurosci 27:5506-5514

Cools R, Frank MJ, Gibbs SE, Miyakawa A, Jagust W, D’Esposito M (2009) Striatal dopamine predicts outcome-specific reversal learning and its sensitivity to dopaminergic drug administration. $\mathrm{J}$ Neurosci 29:1538-1543

Ding JB, Guzman JN, Peterson JD, Goldberg JA, Surmeier DJ (2010) Thalamic gating of corticostriatal signaling by cholinergic interneurons. Neuron 67:294-307

Dodds CM, Muller U, Clark L, van Loon A, Cools R, Robbins TW (2008) Methylphenidate has differential effects on blood oxygenation level-dependent signal related to cognitive subprocesses of reversal learning. J Neurosci 28:5976-5982

Duann JR, Ide JS, Luo X, Li CS (2009) Functional connectivity delineates distinct roles of the inferior frontal cortex and presupplementary motor area in stop signal inhibition. J Neurosci 29:10171-10179

Eickhoff SB, Paus T, Caspers S, Grosbras MH, Evans AC, Zilles K, Amunts K (2007) Assignment of functional activations to probabilistic cytoarchitectonic areas revisited. NeuroImage 36:511-521

Floel A, de Vries MH, Scholz J, Breitenstein C, Johansen-Berg H (2009) White matter integrity in the vicinity of Broca's area predicts grammar learning success. NeuroImage 47:1974-1981

Forstmann BU, Jahfari S, Scholte HS, Wolfensteller U, van den Wildenberg WP, Ridderinkhof KR (2008) Function and structure of the right inferior frontal cortex predict individual differences in response inhibition: a model-based approach. J Neurosci 28:9790-9796

Friston KJ, Buechel C, Fink GR, Morris J, Rolls E, Dolan RJ (1997) Psychophysiological and modulatory interactions in neuroimaging. NeuroImage 6:218-229

Friston KJ, Rotshtein P, Geng JJ, Sterzer P, Henson RN (2006) A critique of functional localisers. NeuroImage 30:1077-1087

Gazzaley A, Rissman J, Desposito M (2004) Functional connectivity during working memory maintenance. Cogn Affect Behav Neurosci 4:580-599

Gibbs SE, D'Esposito M (2005a) Individual capacity differences predict working memory performance and prefrontal activity following dopamine receptor stimulation. Cogn Affect Behav Neurosci 5:212-221

Gibbs SEB, D'Esposito M (2005b) A functional MRI study of the effects of bromocriptine, a dopamine receptor agonist, on component processes of working memory. Psychopharmacology 180:644-653

Goto Y, Grace AA (2005) Dopaminergic modulation of limbic and cortical drive of nucleus accumbens in goal-directed behavior. Nat Neurosci 8:805-812

Hampshire A, Duncan J, Owen AM (2007) Selective tuning of the blood oxygenation level-dependent response during simple target detection dissociates human frontoparietal subregions. J Neurosci 27:6219-6223

Hazy TE, Frank MJ, O'Reilly RC (2007) Towards an executive without a homunculus: computational models of the prefrontal cortex/basal ganglia system. Philos Trans R Soc Lond B Biol Sci 362:1601-1613

Honey GD, Suckling J, Zelaya F, Long C, Routledge C, Jackson S, Ng V, Fletcher PC, Williams SC, Brown J, Bullmore ET (2003) Dopaminergic drug effects on physiological connectivity in a human cortico-striato-thalamic system. Brain 126:1767-1781

Jocham G, Klein TA, Neumann J, von Cramon DY, Reuter M, Ullsperger M (2009) Dopamine DRD2 polymorphism alters reversal learning and associated neural activity. J Neurosci 29:3695-3704

Kimberg DY, Desposito M, Farah MJ (1997) Effects of bromocriptine on human subjects depend on working memory capacity. Neuroreport 8:3581-3585
Kriegeskorte N, Simmons WK, Bellgowan PS, Baker CI (2009) Circular analysis in systems neuroscience: the dangers of double dipping. Nat Neurosci 12:535-540

Kriegeskorte N, Lindquist MA, Nichols TE, Poldrack RA, Vul E (2010) Everything you never wanted to know about circular analysis, but were afraid to ask. J Cereb Blood Flow Metab 30:1551-1557

Krugel LK, Biele G, Mohr PN, Li SC, Heekeren HR (2009) Genetic variation in dopaminergic neuromodulation influences the ability to rapidly and flexibly adapt decisions. Proc Natl Acad Sci U S A 106:17951-17956

Mehta MA, Riedel WJ (2006) Dopaminergic enhancement of cognitive function. Curr Pharm Des 12:2487-2500

Moss J, Bolam JP (2010) The relationship between dopaminergic axons and glutamatergic synapses in the striatum: structural considerations. In: Iversen L, Iversen SD, Bunnett SB, Bjorklund A (eds) Dopamine handbook. Oxford University Press, New York

Nagano-Saito A, Leyton M, Monchi O, Goldberg YK, He Y, Dagher A (2008) Dopamine depletion impairs frontostriatal functional connectivity during a set-shifting task. J Neurosci 28:3697-3706

Neubert FX, Mars RB, Buch ER, Olivier E, Rushworth MF (2010) Cortical and subcortical interactions during action reprogramming and their related white matter pathways. Proc Natl Acad Sci U S A 107:13240-13245

Noudoost B, Moore T (2011) Control of visual cortical signals by prefrontal dopamine. Nature 474:372-375

Petrides M, Pandya DN (2009) Distinct parietal and temporal pathways to the homologues of Broca's area in the monkey. PLoS Biol 7:e1000170

Rorden C, Karnath HO, Bonilha L (2007) Improving lesion-symptom mapping. J Cogn Neurosci 19:1081-1088

Seamans JK, Yang CR (2004) The principal features and mechanisms of dopamine modulation in the prefrontal cortex. Prog Neurobiol 74:1-58

Stelzel C, Basten U, Montag C, Reuter M, Fiebach CJ (2010) Frontostriatal involvement in task switching depends on genetic differences in $\mathrm{D}_{2}$ receptor density. J Neurosci 30:14205-14212

Studholme C, Constable RT, Duncan JS (2000) Accurate alignment of functional EPI data to anatomical MRI using a physics-based distortion model. IEEE Trans Med Imaging 19:1115-1127

Tuch DS, Salat DH, Wisco JJ, Zaleta AK, Hevelone ND, Rosas HD (2005) Choice reaction time performance correlates with diffusion anisotropy in white matter pathways supporting visuospatial attention. Proc Natl Acad Sci U S A 102:12212-12217

Tzourio-Mazoyer N, Landeau B, Papathanassiou D, Crivello F, Etard O, Delcroix N, Mazoyer B, Joliot M (2002) Automated anatomical labeling of activations in SPM using a macroscopic anatomical parcellation of the MNI MRI single-subject brain. NeuroImage 15:273-289

van der Schaaf ME, van Schouwenburg MR, Geurts DE, Schellekens AF, Buitelaar JK, Verkes RJ, Cools R (2012) Establishing the dopamine dependency of human striatal signals during reward and punishment reversal learning. Cereb Cortex. doi:10.1093/cercor/bhs344

van Schouwenburg MR, den Ouden HEM, Cools R (2010) The human basal ganglia modulate frontal-posterior connectivity during attention shifting. J Neurosci 30:9910-9918

Visser E, Qin S., Zwiers M. (2010) EPI distortion correction by constrained nonlinear coregistration improves group fMRI. Presented at Joint Annual Meeting ISMRM-ESMRMB, Stockholm, Sweden

Wallace DL, Vytlacil JJ, Nomura EM, Gibbs SE, D’Esposito M (2011) The dopamine agonist bromocriptine differentially affects frontostriatal functional connectivity during working memory. Front Hum Neurosci 5:32

Zandbelt BB, Vink M (2010) On the role of the striatum in response inhibition. PLoS One 5:e13848

Zwiers MP (2010) Patching cardiac and head motion artefacts in diffusion-weighted images. NeuroImage 53:565-575 